\title{
Developmental changes in the speed and effects of acoustic and semantic encoding
}

\author{
JEFFREY BISANZ \\ University of Alberta, Edmonton, Alberta, T6G 2E9, Canada \\ ROBERT KAIL \\ Purdue University, Lafayette, Indiana 47907 \\ JAMES W. PELLEGRINO \\ University of California, Santa Barbara, California 93106
}

and

\author{
ALEXANDER W. SIEGEL \\ University of Houston, Houston, Texas 77004
}

\begin{abstract}
An incidental learning procedure was used with 8-, 11-, and 19-year-olds to identify processing changes that might underlie a developmental shift in feature encoding. Presentation of each of 32 words was accompanied by a question designed to induce either acoustic or semantic encoding. Tests of recall and recognition followed an intervening task. Acoustic encoding was faster than semantic encoding at all ages, but this difference decreased with age. Semantic encoding resulted in greater recall and recognition accuracy than acoustic encoding only for adults. It is argued that decreases in the difference between acoustic and semantic encoding speeds could contribute to the encoding shift effect. The retention data are interpreted as support for the notion that the encoding shift effect may also be due, in part, to memory processes other than encoding. Changes in encoding speeds and in memory processes related to retrieval are considered as complementary aspects of memory development.
\end{abstract}

Considerable research has been directed toward understanding developmental changes in encoding (Kail \& Siegel, 1977). One suggestion has been that semantic encoding becomes more prevalent with age relative to acoustic encoding (Underwood, 1969). In the present study, two factors were examined that might account, in part, for this shift in encoding and that might also have more general implications for memory development.

The first factor concerns whether an individual successfully encodes semantic and acoustic features of words. Semantic encoding might become increasingly dominant because the likelihood of successful semantic encoding increases with age, relative to the likelihood of successful acoustic encoding. The question then becomes one of identifying developmental changes that

This research was conducted while the authors were affiliated with the University of Pittsburgh. It was supported by the Learning Research and Development Center of the University of Pittsburgh, which is funded in part by the National Institute of Education, U.S. Department of Health, Education, and Welfare; support also came from NICHHD Grant HD-09694 to A. W. Siegel. We are grateful to Gay Bisanz and Susan Goldman for comenting on a previous draft, to Jean Kell, Mary Ficcardi, and John Cameron for serving as experimenters, and to the staff and students of the Weil Elementary School for their cooperation. Reprint requests may be addressed to J. Bisanz, Department of Psychology, University of Alberta, Edmonton, Alberta T6G 2E9, Canada. might contribute to these changing likelihoods. One hypothesis involves changes in encoding speeds (cf. Kail \& Siegel, 1977). Encoding is a time-dependent process that is limited in many situations by the rate at which information is encountered. Encoding processes that require more time than others are less likely to be completed successfully in a fixed and brief period. Of particular concern is the difference between acoustic and semantic encoding latencies and whether this difference changes with age. Adults encode acoustic information about $60 \mathrm{msec}$ faster than semantic information (Craik \& Tulving, 1975). If the difference in encoding latencies is larger for younger children, then they should be less likely than adults to encode semantic features as opposed to acoustic features, at least in the limited time periods characteristic of most studies. To test this hypothesis, it is necessary to measure the time required for acoustic and for semantic encoding. If the temporal difference between acoustic and semantic encoding decreases with age, then changes in encoding speeds may underlie the encoding-shift phenomenon.

The second factor concerns the role of memory processes that occur after initial encoding. It is possible that the consequences of semantic vs. acoustic encodings vary independently of changes in encoding per se. Even if acoustic and semantic encodings were identical in younger and older children, semantic encoding might become more effective with age for retention than 
acoustic encoding. For example, susceptibility to interference (or rate of decay) might decrease rapidly with age for semantic information but not for acoustic information, or retrieval processes for acoustic and semantic information might change differentially with age. Either of these explanations could account for the way in which semantic encoding becomes relatively more effective for retention, yet neither requires that encoding per se change with age. To determine if memory processes other than encoding may be, in part, responsible for the encoding-shift effect, it is necessary to ensure that semantic or acoustic features are encoded initially, and then to assess the memorial effects of these different encodings. If retention based on semantic features becomes greater with age relative to retention based on acoustic features, then memory processes other than initial encoding may account for results typically attributed to developmental changes in encoding.

The present study was designed to determine (1) if the difference in speeds between acoustic and semantic encoding changes with age, and (2) if retention based on semantic encoding increases with age relative to retention based on acoustic encoding. Procedures developed by Craik and Tulving (1975) were used because they ensure that a particular feature of a word has actually been encoded, and because they permit measurement of encoding speeds for different types of features.

\section{METHOD}

\section{Subjects and Design}

Fortyeight subjects, 8 males and 8 females at each of three age levels, were tested individually. Median ages for the three groups were (in years:months) $8: 10,11: 10$, and 19:4.

The experiment consisted of three parts: (1) presentation of questions and words, (2) recall, and (3) forced-choice recognition. Combining two within-subjects factors, type of encoding (acoustic, semantic) and decision (yes, no), produced four types of processing that were induced with questions during the first part of the study. The recognition task additionally involved the between-subjects factor of test bias. ${ }^{1}$

\section{Materials}

Thirty-two target words, two from each of 16 semantic categories, were selected from children's category norms (Posnansky, 1974). The words, predominantly one-syllable nouns, were presented in a fixed order. Stimuli for the forcedchoice recognition task consisted of 32 word pairs, each pair consisting of a target and an alternative, arranged horizontally.

\section{Procedure}

Each of 32 words was projected onto a screen for 2.5 sec. Presentation of each word was preceded by a question that required either acoustic or semantic encoding of the word. Acoustic encoding questions were of the form, "Does this rhyme with ? "Semantic encoding questions were of the form, "Is this _ ? ", with reference to a category label (e.g., "a fruit") or to a description (e.g., "something a person wears"). Four lists of such questions were constructed and used in a counterbalanced fashion across subjects so that each word was the focus of each of the four types of questions (acousticyes, acoustic-no, semantic-yes, semantic-no) equally often.
Subjects were not asked to memorize the words. When the word was presented, they were to respond "yes" or "no" by pressing one of two response buttons with the index finger of the preferred hand. Response choice and latency were recorded. Positions of the "yes" and "no" buttons were counterbalanced across subjects. Presentation of words was preceded by 16 practice trials in which subjects were familiarized with the procedure and apparatus.

After an intervening task lasting about $5 \mathrm{~min}$, subjects were asked to recall aloud as many words as possible. The forcedchoice recognition test followed immediately. The 32 pairs of words were projected individually onto a screen, and subjects were told to press the button corresponding to the word they had seen before. Testing required about $30 \mathrm{~min}$.

\section{RESULTS AND DISCUSSION}

\section{Initial Encoding Latencies}

For each subject, a median reaction time (RT) was computed for the correct responses to each of the four types of questions. Because RT variances differed across ages, a separate analysis of variance was performed at each age level on the within-subjects factors of type of encoding (acoustic, semantic) and decision (yes, no). Acoustic encoding was more rapid than semantic encoding at all age levels, although the magnitude of the difference decreased with age. The difference between acoustic and semantic encoding times was $130 \mathrm{msec}$ for 8-year-olds, $50 \mathrm{msec}$ for 11-year-olds, and $49 \mathrm{msec}$ for adults $[\mathrm{Fs}(1,15)=8.73,6.64,4.92$, and $\mathrm{ps}<.05]$. "Yes" decisions were more rapid than "no" decisions, and again, the difference decreased with age. The yes-no difference was $186 \mathrm{msec}$ for 8-year-olds $[\mathrm{F}(1,15)=6.94$, $\mathrm{p}<.025], 61 \mathrm{msec}$ for 11 -year-olds $[\mathrm{F}(1,15)=4.48$, $\mathrm{p}=.05]$, and only $24 \mathrm{msec}$ for adults $[\mathrm{F}(1,15)=2.49$, $\mathrm{p}>.10]$. All Encoding by Decision interactions were nonsignificant.

For adults, semantic processing requires more time than does acoustic processing (e.g, Craik \& Tulving, 1975), while latency differences between "yes" and "no" decisions have been found less frequently. The unique finding of the present study was that both types of latency differences-semantic minus acoustic and no minus yes-were pronounced in 8-year-olds and decreased with age. With the high-frequency words used in this study, the semantic-acoustic difference reached an asymptote by 11 years, while the no-yes difference continued to decrease to a negligible level in early adulthood. ${ }^{2}$ Thus, there was a convergence with age in the speeds with which the various encodings occurred.

\section{Recall Accuracy}

Proportions of correct recall for each subject were computed on the basis of items responded to correctly in the presentation phase. A 3 (age) by 2 (type of encoding) by 2 (decision) analysis of variance with repeated measures on the last two factors revealed that subjects recalled semantically processed items more often than acoustically processed items $[F(1,45)=18.57$, $\mathrm{p}<.001]$. Words involved in the "yes" decisions were 
recalled more often than those in "no" decisions $[F(1,45)=11.30, p<.005]$. Accuracy of recall increased with age $[F(2,45)=9.64, p<.001]$.

More important than the main effects, however, were two interactions involving age. First, the Age by Type of Encoding interaction (Figure 1) was significant $[F(2,45)=4.02, p<.025]$. Tests of simple main effects revealed that the difference between recall of semantically and acoustically processed items occurred with adults $(p<.001)$, but not with the two groups of children ( $\mathrm{ps}>.10)$. Second, the Age by Decision interaction was significant $[F(2,45)=4.58, p<.025]$. Tests of simple effects demonstrated that recall of "yes" items was greater than "no" items only for adults $(\mathrm{p}<.001)$. All values represented in Figure 1 are greater than zero (ps $<.02$ ).

Thus, "yes" decisions produced greater recall than "no" decisions in adults, but not in children. For "yes" decisions, the question accompanying presentation of the target word is presumed to facilitate later recall because both question and word can be integrated into a single elaborated encoding for which the question can serve as a redintegrative cue (Craik \& Tulving, 1975). For "no" decisions, the question and target word cannot be integrated; consequently, the question cannot serve as a redintegrative cue. The fact that the difference in recall resulting from "yes" and "no" decisions increased with age implies that young children are less likely either (1) to spontaneously integrate the question and target word in a single encoding or (2) to use contextual information to aid retrieval.

As in previous studies with adults, semantic encoding produced higher levels of recall than did acoustic encoding. However, the nature of initial encoding (semantic vs. acoustic) had no effect on recall by 8 - and 11-yearolds. The implications of this result are considered below.

\section{Recognition Accuracy}

Only words judged correctly in the presentation phase were considered in computing proportion of

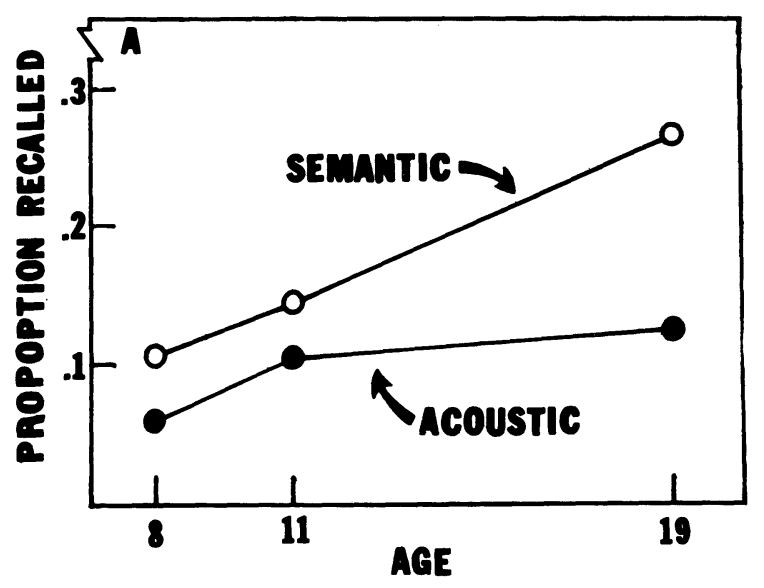

Figure 1. Mean proportion of words recalled as a function of age and type of encoding.

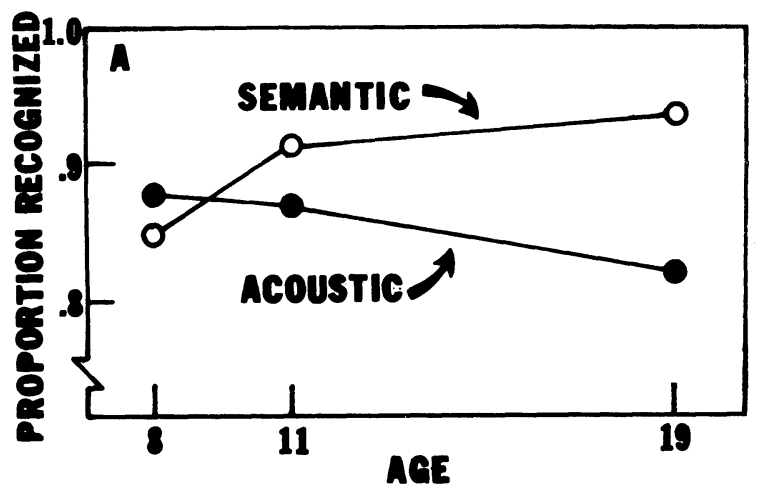

Figure 2. Mean proportion of words recognized correctly as a function of age and type of encoding.

correct recognition. A 3 (age) by 2 (test bias) by 2 (type of encoding) by 2 (decision) analysis of variance was performed on these data. Semantically processed words were recognized more accurately than acoustically processed words $[\mathrm{F}(1,42)=7.57, \mathrm{p}<.01]$, and words involved in "yes" decisions were recognized more accurately than those in "no" decisions $[\mathrm{F}(1,42)=9.21$, $\mathrm{p}<.005]$. Age did not interact with decision, but the Age by Type of Encoding interaction was significant $[F(2,42)=7.98, p<.005$ (see Figure 2) $]$. The difference between correct recognition of semantically and acoustically processed items increased with age. Tests of simple main effects showed that this difference was highly significant for adults $(p<.001)$, marginally significant for 11 -year-olds $(p<.10)$, and nonsignificant for the 8-year-olds $(p>.10)$. No other interactions or main effects were significant.

As with recall, the relationship between initial processing and recognition accuracy changed with age. For adults, semantic processing produced higher recognition accuracy than did acoustic processing, an effect that was only marginally significant for sixth-graders. For younger children, accuracy was unaffected by initial processing.

\section{GENERAL DISCUSSION}

The purpose of the present study was to investigate possible processes underlying the way in which semantic encoding becomes increasingly important, relative to acoustic encoding, with age. The most pertinent outcomes were (1) convergence with age in the latencies of acoustic and semantic encoding, and (2) divergence with age in the memorial consequences of these encodings.

Based on the latency data, it appears that changes in the speeds of acoustic and semantic encodings could have contributed to the encoding-shift effect found in previous research. Acoustic processing of words will be completed more often than semantic processing because it occurs more rapidly than semantic processing. The difference between semantic and acoustic encoding speeds was especially large for younger children, and consequently, younger children should be less likely to complete semantic encoding within a fixed period of time. As this difference in encoding speeds declines with age, the relative likelihood of successful semantic encoding should increase. This could account in part, for age-related increases in the importance of semantic encoding for retention. 
Given the retention data, it can also be argued that results consistent with the idea of an encoding shift may be due to changes in memory processes other than initial encoding. Interactions between age and type of encoding were found even though initial encoding was controlled, at least to the extent that minimal encodings of certain features were elicited. Semantic encoding was found to facilitate retention only for adults. For children, not only did semantic encoding require more time than acoustic encoding, but the memorial benefits of semantic encoding were small and nonsignificant as compared with acoustic encoding. It should be emphasized that we are not suggesting that semantic encoding cannot facilitate young children's retention, since such facilitation has been demonstrated previously (e.g., Murphy \& Brown, 1975). Rather, it appears that the memorial effects of semantic encoding become more pronounced with age relative to the effects of acoustic encoding. ${ }^{3}$ The reason for this change may be that older individuals create more detailed and distinctive semantic representations (even when answering very simple questions), thereby reducing the effects of interference or decay in retention (cf. Wickelgren, 1975).

More generally, both the encoding time and retention data from the present study may reflect the fact that knowledge of the acoustic and semantic features of language develops at different rates. Phonological development occurs rapidly during the preschool years (Menyuk, 1971). By 8 years of age, acoustic knowledge may already be stable and well organized; future linguistic interactions probably require little, if any, further development. In contrast, semantic knowledge develops over a much longer period of time (e.g., Anglin, 1970). If development is defined by greater organization and differentiation of memory and by a concommitant increase in the efficiency of memory processes, then access to and use of semantic information should continue to improve relative to access to and use of acoustic information, especially after acoustic knowledge has reached an asymptote. The decline with age in the relative latency of encoding semantic features and the increase with age in the relative benefits of semantic encoding for retention appear to reflect such a pattern of development. Thus, changes in encoding speeds and in processes related to storage and retrieval can be viewed as complementary aspects of memory development.

In summary, previous retention data have frequently, but ambiguously, been interpreted as evidence for a developmental shift in the encoding of acoustic and semantic features of words. These results may have been a function of (1) changes in acoustic and semantic encoding speeds and/or (2) changes in processes involved in retaining acoustic and semantic information. Both types of change are likely to reflect more basic patterns in the development of acoustic and semantic knowledge. The important task now is not to understand the encoding shift effect per se, but rather to identify the complex relationships between the development of encoding skills and changes in the organization and use of acoustic and semantic information.

\section{REFERENCES}

Anglin, J. M. The growth of word meaning. Cambridge, Mass: M.I.T. Press, 1970.

Craik, F. I. M., \& Tulving, E. Depth of processing and the retention of words. Journal of Experimental Psychology: General, 1975, 1, 268-294.

Geis, M. F., \& HALL, D. M. Encoding and incidental memory in children. Journal of Experimental Child Psychology, 1976, 22, 58-66.

KaIL, R. V., \& Siegel, A. W. The development of mnemonic encoding in children: From perception to abstraction. In R. V. Kail \& J. W. Hagen (Eds.), Perspectives on the development of memory and cognition. Hillsdale, N.J: Erlbaum, 1977.

MeNYuK, P. The acquisition and development of language. Englewood Cliffs, N.J: Prentice-Hall, 1971.

Murphy, M. D., \& Brown, A. L. Incidental learning in preschool children as a function of level of cognitive analysis. Journal of Experimental Child Psychology, 1975, 19, 509-523.

Posnansky, C. G. Category norms of children. Behavior Research Methods \& Instrumentation, 1974, 6, 373.

Unde rwood, B. J. Attributes of memory. Psychological Review, 1969, 76, 559-573.

Wickelgren, W. A. More on the long and short of memory. In D. Deutsch \& J. A. Deutsch (Eds.), Short-term memory. New York: Academic Press, 1975.

\section{NOTES}

1. In the biased condition, recognition alternatives were related either acoustically or semantically to the target words; in the unbiased condition, the alternatives were unrelated. None of the effects associated with bias were significant, so these manipulations will not be discussed further.

2. While both the semantic-acoustic and no-yes differences declined substantially with age, it should be noted that they are not demonstrated statistically because of the need to do separate analyses at each age level. Ignoring heterogeneity of variance problems, the semantic-acoustic difference for 8-year-olds was greater than that for the 11- and 18-year-olds combined $[F(1,42)=4.38, p<.05]$, and the Age by Decision interaction was significant $[F(2,42)=3.67, p<.05]$.

3. This is contrary to the conclusion of Geis and Hall (1976), who reported that semantic encoding facilitated recall equally for 7-, 8-, and 11-year-olds. Several differences between our study and theirs preclude a direct comparison. Note, however, that the changes in recall in the present study were found between ages 11 and 19, and thus could not be detected by Geis and Hall, whose oldest subjects were 11 years of age. The boundary conditions within which semantic encoding facilitates retention, relative to other forms of encoding, clearly require further specification.

(Received for publication July 3, 1979.) 